\title{
Dynamic Analysis of a Fractional Order Three Species Interaction Model with Holling Type II Response
}

\author{
A. George Maria Selvam ${ }^{1}$, D.Vignesh ${ }^{2}$ and S. Britto Jacob ${ }^{3}$ \\ Department of Mathematics ${ }^{1,2,3}$, \\ Sacred Heart College(Autonomous), \\ Tirupattur-635601, Vellore. \\ agmshc@gmail.com ${ }^{1}$
}

\begin{abstract}
This paper considers a three species predator-prey model with Holling type II response in fractional order. The model is investigated with the assumption that apex predator consumes both the logistically growing prey and the mesopredators. Stability properties and the existence of periodic solutions are examined for the equilibrium points and the results are strengthened with the numerical simulations. Time evolution of the Lyapunov Exponents of the fractional order system is tabulated and dynamics of the Lyapunov Exponent is discussed with the 2-D plot. Also, the effect of the fractional order on the dynamics of the system is analyzed. Time plots, phase plane graphs and bifurcations diagrams are presented.
\end{abstract}

Index Terms- Fractional differential equation; Discretization; Population Dynamics; Fixed Points; Stability; Bifurcation;

\section{INTRODUCTION}

Fractional calculus has its origin during the $17^{\text {th }}$ century, but it is $20^{\text {th }}$ century when most of the theory applicable to study Fractional calculus has been developed. Despite the long history, motivation for the researchers to work on fractional calculus has been increasing over a decade. Especially, lots of works are carried out on fractional order systems and its applications in various fields and recent developments on the theory on existence and uniqueness, stability conditions of solutions of non-linear FDEs. [[3],[5]]

In discussion of the stability properties of fractional order systems the following lemmas are used.

Lemma 1.1: [[4],[7]] The following linear commensurate fractional - order autonomous system

$$
D^{\alpha} x=A x, x(0)=x_{0}
$$

is asymptotically stable if and only if $|\arg \lambda|>\alpha \frac{\pi}{2}$ is satisfied for all eigenvalues of matrix A. Also, this system is stable if and only if $|\arg \lambda|>\alpha \frac{\pi}{2}$ is satisfied for all eigenvalues $(\lambda)$ of matrix $A$ and those critical eigenvalues which satisfy $|\arg \lambda|=\alpha \frac{\pi}{2}$ have geometric multiplicity one, where $0<\alpha<1$, $x \in R^{n}$ and $A \in R^{n \times n}$

Lemma 1.2: [[4],[7]] The fixed points of the following linear commensurate fractional - order autonomous system is asymptotically stable if all eigenvalues $(\lambda)$ of the Jacobian matrix $\frac{\partial f}{\partial x}$ evaluate at the fixed points satisfy $|\arg \lambda|>\alpha \frac{\pi}{2} \quad$ where $\quad 0<\alpha<1, \quad x \in R^{n} \quad$ and $f: R^{n} \rightarrow R^{n}$ are continuous linear vector functions and the fixed points of this nonlinear commensurate fractional order system are calculated by solving equation $f(x)=0$.

\section{BACKGROUND OF THE MATHEMATICAL MODEL}

Major In this paper, we consider an ecosystem of three species interaction with Holling type II functional response. Prey, Meso predators and Apex predators are modelled based on the assumption that Apex predator survives on predation of both logistically growing prey and Meso predators. Meso predators depends only on prey for its survival.

Main purpose of modelling a ecological system with a functional response is to represent the consumers rate of intake as a function of density of availability of food in that ecosystem. Thus, functional response of Holling type II is introduced to the system to decelerate the rate of intake by the predators (or) the predators food processing capacity is limited.

System of Fractional order differential equations representing the three species interaction is given by

$$
D^{\gamma_{1}} x(t) F^{\alpha} \gamma_{x}\left(\underline{1} A \mathcal{X},\left(t d \partial \partial(\underline{t}) \overline{x_{0}} \frac{a x(t) y(t)}{1+x(t)}-\frac{c x(t) z(t)}{1+x(t)}\right.\right.
$$




\section{Available online at www.ijrat.org}

$$
\begin{aligned}
& D^{\gamma_{2}} y(t)=\frac{a x(t) y(t)}{1+x(t)}-\frac{b y(t) z(t)}{1+y(t)}-d y(t) \\
& D^{\gamma_{3}} z(t)=\frac{c x(t) z(t)}{1+x(t)}+\frac{b y(t) z(t)}{1+y(t)}-e z(t)
\end{aligned}
$$

where $x(t), y(t)$ and $z(t)$ represents the prey density, Meso predator density and Apex predator density respectively with $\gamma_{1}, \gamma_{2}$ and $\gamma_{3}$ being their fractional orders.In Biological populations, all the parameters of the system (1) are positive.

\begin{tabular}{|c|c|}
\hline Symbols & Meaning \\
\hline$r$ & $\begin{array}{c}\text { Growth rate of the prey } \\
\text { Apex predator }\end{array}$ \\
\hline$b$ & $\begin{array}{c}\text { Interaction rate of meso predator and } \\
\text { Natural death rate of the meso predator } \\
\text { predator }\end{array}$ \\
\hline$a$ & $\begin{array}{c}\text { Interaction rate of Prey and meso } \\
\text { predator }\end{array}$ \\
\hline$c$ & $\begin{array}{c}\text { Interaction rate of prey and Apex } \\
\text { patural death rate of the Apex predator }\end{array}$ \\
\hline
\end{tabular}

\section{FIXED POINT AND STABILITY ANALYSIS OF THE SYSTEM (1)}

Displayed The non-negative Axial fixed points of the Fractional order system (1) are

- $E_{0}=(0,0,0)$ (trivial)

- $E_{1}=(1,0,0)$ (Predator free)

- $E_{2}=\left(\frac{e}{c-e}, 0, \frac{r(c-2 e)}{(c-e)^{2}}\right)$ (Non existence of Meso predators )

- $E_{3}=\left(\frac{d}{a-d}, \frac{r(a-2 d)}{(a-d)^{2}}, 0\right)$ (Non existence of Apex Predator)

The Jacobian matrix of the Fractional order system (1) for equilibrium $E=(x, y, z)$ is

$$
J\left(E_{0}\right)=\left[\begin{array}{lll}
r & 0 & 0 \\
0 & -d & 0 \\
0 & 0 & -e
\end{array}\right]
$$

The Eigenvalues of the matrix $J\left(E_{0}\right)$ are $\lambda_{1}=r, \lambda_{2}=$ $-e$ and $\lambda_{3}=-d$

\section{Predator free Equilibrium $\left(E_{1}\right)$}

$$
J\left(E_{1}\right)=\left[\begin{array}{ccc}
-r & -\frac{a}{2} & -\frac{c}{2} \\
0 & \frac{a}{2}-d & 0 \\
0 & 0 & \frac{c}{2}-e
\end{array}\right]
$$

$\lambda_{1}=-r, \lambda_{2}=\frac{c}{2}-e \quad$ and $\quad \lambda_{3}=\frac{a}{2}-d \quad$ are the eigenvalues of the matrix $J\left(E_{1}\right)$.

Meso Predator free Equilibrium $\left(E_{2}\right)$

$$
\begin{aligned}
& J\left(E_{2}\right)= \\
& {\left[\begin{array}{cc}
-2 \frac{e^{2} r}{c(c-e)} & -\frac{a e}{c} \\
0 & \frac{a c^{2} e-2 a c e^{2}+a e^{3}-b r c^{2}+2 b r c e-c^{3} d+2 c^{2} d e-c d e^{2}}{(c-e)^{2} c} \\
\frac{r(c-2 e)}{c} & \frac{b r(c-2 e)}{(c-e)^{2}}
\end{array}\right.}
\end{aligned}
$$

The corresponding eigenvalues are

$$
\begin{aligned}
& \lambda_{1,2}=\frac{-r e^{2} \pm \sqrt{-c^{4} e r+4 c^{3} e^{2} r-5 c^{2} e^{3} r+2 c e^{4} r+e^{4} r^{2}}}{(c-e) c} \text { and } \\
& \lambda_{3}=\frac{a c^{2} e-2 a c e^{2}+a e^{3}-b c^{2} r+2 b c e r-c^{3} d+2 c^{2} d e-d c e^{2}}{c\left(c^{2}-2 c e+e^{2}\right)} .
\end{aligned}
$$

\section{Apex Predator free Equilibrium $\left(E_{3}\right)$}

$$
\begin{aligned}
& J\left(E_{3}\right) \\
& =\left[\begin{array}{ccc}
-2 \frac{d^{2} r}{a(a-d)} & -d & -\frac{c d}{a} \\
\frac{r(a-2 d)}{a} & 0 & -\frac{b r(a-2 d)}{a^{2}-2 a d+a r+d^{2}-2 r d} \\
0 & 0 & F
\end{array}\right]
\end{aligned}
$$

$$
=\left[\begin{array}{c}
r(1-x)-r x-\frac{a y}{1+x}+\frac{a x y}{(1+x)^{2}}-\frac{c z}{1+x}+\frac{c x z}{(1+x)^{2}} \\
\frac{a y}{1+x}-\frac{a x y}{(1+x)^{2}} \\
\frac{c z}{1+x}-\frac{c x z}{(1+x)^{2}}
\end{array}\right.
$$

$$
\begin{aligned}
& -\frac{c x}{1+x} \\
& -\frac{b y}{1+y}
\end{aligned}
$$$$
\frac{b z}{1+y}-\frac{b y z}{(1+y)^{2}} \quad \frac{b y}{1+y}+\frac{c x}{1+x}-e
$$

where

3.1 Jacobian Matrix for the Fixed points

Trivial fixed point $\left(E_{0}\right)$ 
F

$=-\frac{a^{3} e-b r a^{2}-a^{2} c d-2 a^{2} d e+a^{2} e r+2 b r a d+2 a c d^{2}-a c d r+a d^{2} e-2 a d e r-c d^{3}+2 c d^{2} r}{a\left(a^{2}-2 a d+a r+d^{2}-2 r d\right)}$

$\lambda_{1,2}=\frac{-d^{2} r \pm \sqrt{-a^{4} d r+4 a^{3} d^{2} r-5 a^{2} d^{3} r+2 a d^{4} r+d^{4} r^{2}}}{(a-d) a}$ and

$\lambda_{3}$

$=-\frac{a^{3} e-a^{2} b r-a^{2} c d-2 a^{2} d e+a^{2} e r+2 a b d r+2 a c d^{2}-a c d r+a d^{2} e-2 a d e r-c d^{3}+2 c d^{2} r}{a\left(a^{2}-2 a d+a r+d^{2}-2 r d\right)}$ are the eigenvalues of the matrix $J\left(E_{3}\right)$.

\section{NUMERICAL EXAMPLES}

For the parameter values $r=0.35, a=0.27, \quad b=0.01, c=0.5, d=0.2$, $\mathrm{e}=0.02$

and the initial condition is $(0.05,0.02,0.5)$, the
The condition $|\arg \lambda|>\alpha \frac{\pi}{2}$ is satisfied by all the eigen values,thus the system is asymptotically stable.The stability of the system for each species density is explained in Figure (1) and the 2-D and 3-D phase portrait is also given.
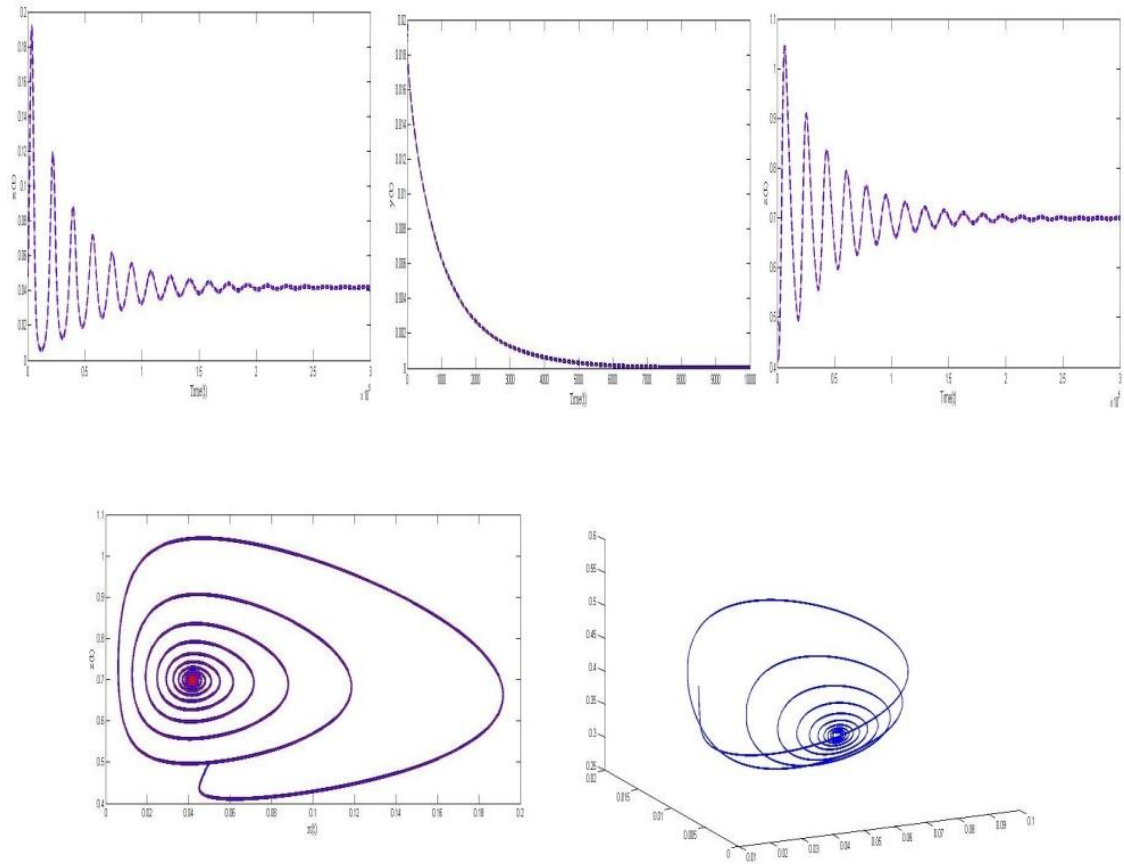

Fig. 1. Time plots and Phase portrait for the system (1).

commensurate fractional order system (1) with order

$$
\gamma_{1}=\gamma_{2}=\gamma_{3}=0.97
$$

has eigen values

$$
\lambda_{1}=-0.0005833333332 \pm 0.08024749045 i
$$
and

$$
\lambda_{3}=-0.1961878472 \text {. }
$$

Figure (2) explains the Limit cycle of the the commensurate fractional order system (1) with order $\gamma_{1}=\gamma_{2}=\gamma_{3}=0.9$

and initial condition is $(0.45,0.35,0.15)$ with parameter values

$$
\begin{gathered}
r=2.99, a=0.82, b=0.64, c=0.2, d=0.18, \\
e=0.4
\end{gathered}
$$



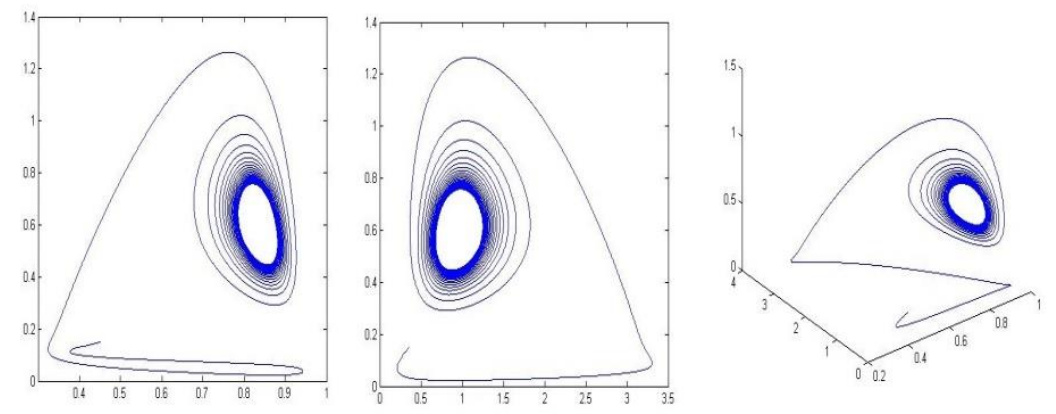

Fig. 2. Limit cycle for the system (1).

\section{DISCRETIZATION OF THE SYSTEM (1)}

Applying piecewise constant arguments methods [1] to the commensurate fractional order system (1) of order $\gamma$, the discretized fractional order system is of the form

$$
\begin{aligned}
& x(t+1)=x(t)+\mathrm{L}\left[r(1-x(t)) x(t)-\frac{a x(t) y(t)}{1+x(t)}-\frac{c x(t) z(t)}{1+x(t)}\right] \\
& y(t+1)=y(t)+\mathrm{L}\left[\frac{a x(t) y(t)}{1+x(t)}-\frac{b y(t) z(t)}{1+y(t)}-d y(t)\right] \\
& z(t+1)=z(t)+\mathrm{L}\left[\frac{c x(t) z(t)}{1+x(t)}+\frac{b y(t) z(t)}{1+y(t)}-e z(t)\right]
\end{aligned}
$$

(2)

where $\gamma$ is the fractional order, $L=\frac{h^{\gamma}}{\Gamma(1+\gamma)}$ and $h$ is the step size of discretization

\section{BIFURCATION OF THE SYSTEM (2)}

The Bifurcation is the study of the changes that occur in the system when a value of the parameter is varied. Bifurcation diagrams helps in understanding the chaotic nature of the system.[2] In this paper, the bifurcation of the system (3) is obtained for the growth rate of the prey $(r)$ and the order $(\gamma)$ of the system (2).

\subsection{Growth rate as Bifurcation parameter}

For the fixed values of parameters $h=1.13, \gamma=$ $0.85, a=0.82, b=0.64, c=0.2, d=0.18, e=0.4$, varying the growth rate $(r)$ in the range 0 to 3 , initial condition is $(0.45,0.35,0.15)$, the bifurcation diagrams in the three dimensions are obtained for the bifurcation parameter as shown in the Figure(3).
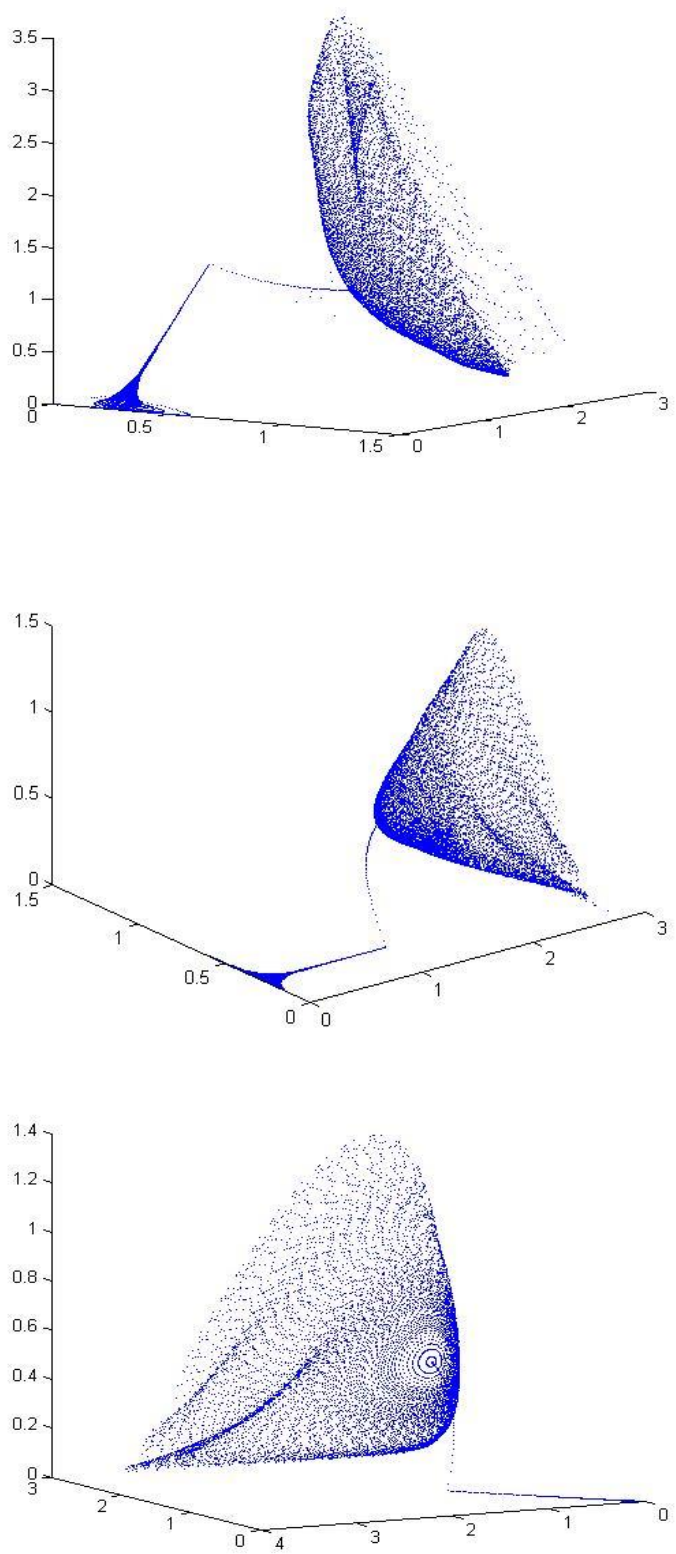

Figure 3: Bifurcation Parameter (r) 


\section{Available online at www.ijrat.org}

\subsection{Fractional order as Bifurcation Parameter}

Considering the range of the Bifurcation parameter range in between 0 to 2, the diagram in Figure (4) is simulated for the fixed parametric values $h=1.13, r=1.99, a=0.82, b=0.64, c=0.2$,

$$
d=0.28, e=0.4
$$

with initial condition $(0.45,0.35,0.15)$.
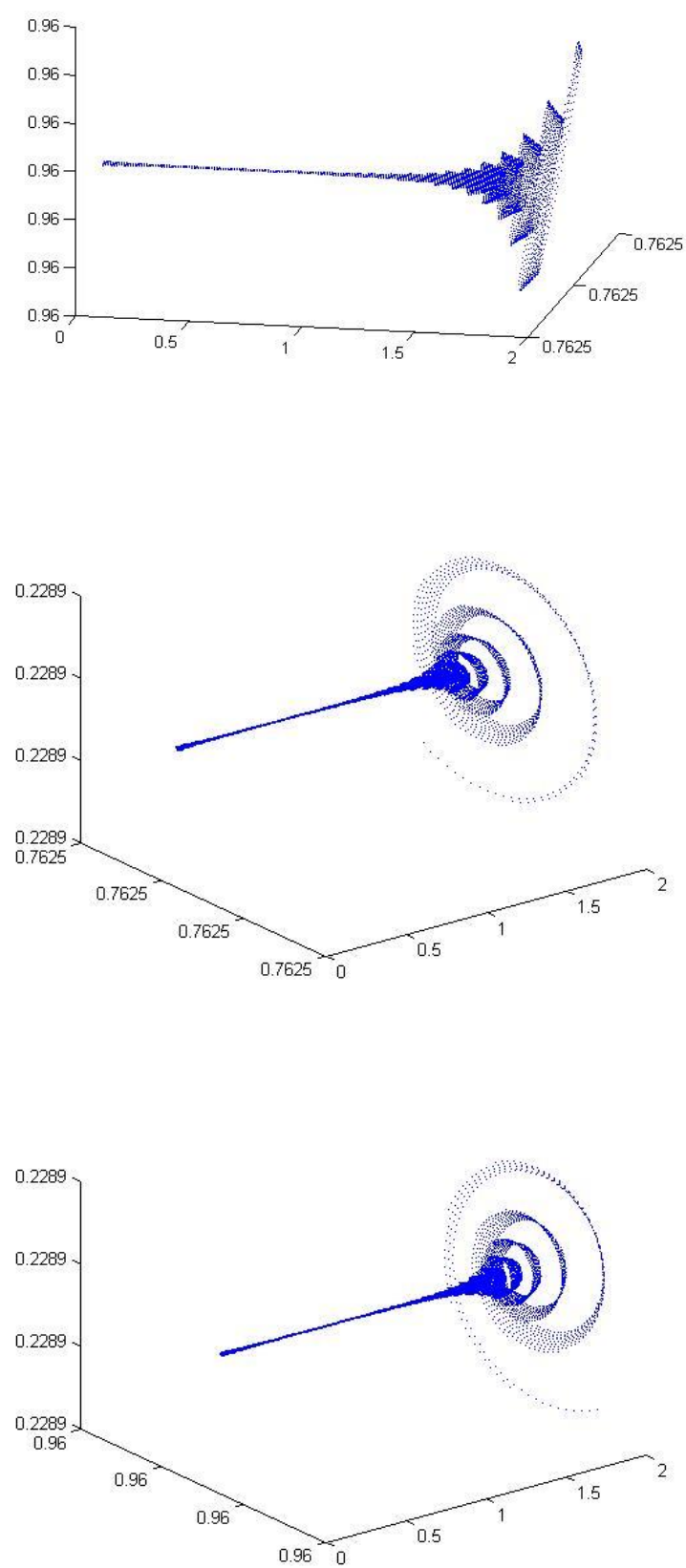

Figure 4: Bifurcation Parameter $(\gamma)$

\section{LYAPUNOV EXPONENTS}

Any two orbits starting from nearby initial points may either converge or diverge.[6] The measure of their average rate of convergence or divergence is the Lyapunov exponents. In this paper, we obtain the Lyapunov exponents as a function of time and the values used for plotting is provided in table [1].

Figure (6) describes the Lyapunov exponents of the fractional order system (1) with commensurate fractional order 0.92 with the parameters $r=0.75, a=0.8, b=51, c=0.64, d=0.1, e=0.4$ and initial conditions $(0.008,0.009,0.007)$.

Table 1. Time Evolution of LE.

\begin{tabular}{|c|c|c|c|}
\hline Time & LE1 & LE2 & LE3 \\
\hline 10 & 0.1729 & -0.0940 & -0.2806 \\
\hline 20 & 0.1294 & -0.1132 & -0.3444 \\
\hline 30 & 0.0652 & -0.1007 & -0.2830 \\
\hline 40 & 0.0532 & -0.0843 & -0.2326 \\
\hline 50 & 0.0375 & -0.0708 & -0.2192 \\
\hline 60 & 0.0352 & -0.0770 & -0.1952 \\
\hline 70 & 0.0282 & -0.0620 & -0.1889 \\
\hline 80 & 0.0234 & -0.0664 & -0.1763 \\
\hline 90 & 0.0202 & -0.0561 & -0.1712 \\
\hline 100 & 0.0133 & -0.0548 & -0.1647 \\
\hline 110 & 0.0097 & -0.0479 & -0.1600 \\
\hline 120 & 0.0033 & -0.0434 & -0.1566 \\
\hline 130 & 0.0008 & -0.0403 & -0.1527 \\
\hline 140 & 0.0014 & -0.0408 & -0.1505 \\
\hline 150 & 0.0023 & -0.0423 & -0.1475 \\
\hline 160 & 0.0019 & -0.0412 & -0.1457 \\
\hline 170 & 0.0007 & -0.0408 & -0.1436 \\
\hline 180 & -0.0008 & -0.0387 & -0.1420 \\
\hline 190 & -0.0032 & -0.0367 & -0.1405 \\
\hline 200 & -0.0057 & -0.0340 & -0.1391 \\
\hline
\end{tabular}

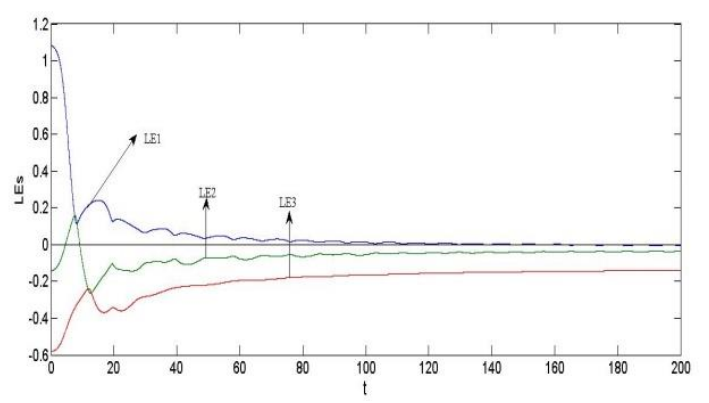

Figure 5: Lyapunov Exponent of the system (1) 


\section{Available online at www.ijrat.org}

\section{CONCLUSION}

The paper investigated the stability properties of the non-linear fractional order dynamical system with holling type II response. Time plots and phase portraits are provided to explain the dynamics of the system. The bifurcation of the system in its equivalent discretized form is analyzed with 2-D and 3-D bifurcation diagrams. Growth parameter and fractional order considered as bifurcation parameter are used to study the chaotic properties. The Lyapunov exponents are calculated and plotted against time. The fractional order systems clearly exhibits rich dyanmics of a non linear dynamical system.

\section{REFERENCES}

[1] Elsadany (2015), Dynamical behaviors of fractional-order lotka-volterra predator-prey model and its discretization. J. Appl. Math. Comput., 49:269-283.

[2] Elsadany, A.-E. A. (2012), Chaos and bifurcation of a nonlinear discrete prey-predator system. Computational Ecology and Software, 2(3):169180.

[3] Guo, Y. (2014). The stability of solutions for a fractional predator-prey system. Abstract and Applied Analysis, 2014:7 pages.

[4] Petras, I. (2010). Fractional order nonlinear systems - modeling, analysis and simulation. Springer International Edition.

[5] Selvam, A. G. M. (2015). Dynamics in a fractional order prey - predator interactions.Mathematical Modelling and Applied Computing, 6(1):1-6.

[6] Skokos, C. (2010). The lyapunov characteristic exponents and their computation. Lecture Notes in Physics 790, pages 63-135.

[7] Zhou, P. (2012). Control and synchronization of the fractional - order lorenz chaotic system via fractional - order derivative. Mathematical Problems in Engineering, Hindawi Publishing, 2012:1-14. 